\title{
Polysulfide anions as visible light photoredox catalysts for aryl cross-couplings
}

Haoyu Li ${ }^{1}$, Xinxin Tang*2, Jia Hao Pang*1, Xiangyang Wu ${ }^{1}$, Edwin K. L. Yeow ${ }^{1}$, Jie Wu ${ }^{2}$, Shunsuke Chiba ${ }^{1 \dagger}$

${ }^{1}$ Division of Chemistry and Biological Chemistry, School of Physical and Mathematical Sciences, Nanyang Technological University, 637371, Singapore.

${ }^{2}$ Department of Chemistry, National University of Singapore, 3 Science Drive 3, 177543, Singapore.

*These authors contributed equally to this work.

†Correspondence to: shunsuke@ntu.edu.sg

ABSTRACT: Polysulfide anions are endowed with unique redox properties, attracting considerable attentions for their applications in alkali metals-sulfur batteries. However, employment of these anionic species in redox catalysis for small molecule synthesis remains underdeveloped due to their poor electrochemical potential in the ground state, whereas some of them are characterized by photo-absorptions in visible spectral regions. Herein, we disclose the use of polysulfide anions as visible light photoredox catalysts for aryl cross-coupling reactions. The reaction design enables single-electron-reduction of aryl halides upon photo-excitation of tetrasulfide dianions $\left(\mathrm{S}_{4}{ }^{2-}\right)$. The resulting aryl radicals are engaged in (hetero)biaryl cross-coupling, borylation, and hydrogenation in a redox catalytic regime involving $\mathrm{S}_{4}{ }^{-} / \mathrm{S}_{4}{ }^{2-}$ and $\mathrm{S}_{3}{ }^{-} / \mathrm{S}_{3}{ }^{2-}$ redox couples.

Main Text: Visible-light photoredox catalysis has advanced the-state-of-the-art in chemical synthesis, enabling to harness low energy visible light to productively drive various types of useful molecular transformations (1-5). Homogeneous photocatalysts such as ruthenium/iridium-based polypyridyl complexes or organic dyes could be excited under irradiation of visible light, inducing single-electron-transfer (SET) to or from organic substrates to provide reactive open-shell radical intermediates. Employment of heterogeneous semiconductors as a redox active chromophore for synthesis of complex molecules has recently offered another contemporary trend in photoredox catalysis (6-8). Nonetheless, further development of new photocatalysts based on inexpensive and abundant elements that can perform productive bond formation processes in a highly efficient fashion is of prominent interests.

Sulfur is known to form various catenated homoatomic polysulfide dianions $\mathrm{S}_{\mathrm{x}}{ }^{2-}$ (typically, $\mathrm{x}=2-8$ ) and a persistent radical anion $\mathrm{S}_{3}{ }^{-}$which is known as a blue chromophore in ultramarine blues $(9,10)$. In seeking for the development of alkali metals-sulfur batteries, chemical reactivity and redox characters of polysulfide anions have been elucidated in details (11). Polysulfide anions undergo complicated redox, dissociative and disproportionation processes in the solution states to afford an equilibrium mixture of multiple polysulfide anions and their steady states depend majorly on the solvents. The in situ spectroelectrochemical studies on the reduction of octasulfur $\left(\mathrm{S}_{8}\right)$ identified the ground state redox couples of $\mathrm{S}_{3}{ }^{-} / \mathrm{S}_{3}{ }^{2-}$ and $\mathrm{S}_{4}{ }^{-} / \mathrm{S}_{4}{ }^{2-}$, and their electrochemical potentials are estimated as around $-1.35 \mathrm{~V}$ and $-0.85 \mathrm{~V}$, respectively, versus saturated calomel electrode (SCE) in dimethylformamide (DMF) (12-14) (Fig. 1A). However, employment of these homoatomic sulfide anions in redox catalysis that engages organic electrophores in the radical-mediated reactions remains 
unmet challenge. Nonetheless, their electrochemical potentials dictate that they are incapable of inducing single-electron-reduction of unactivated organic electrophores of highly negative reduction potentials such as aryl halides $\left[E_{\text {red }}<-1.9 \mathrm{~V}\right.$ (vs SCE)] (15). On the other hand, these species show characteristic absorbance in the ultraviolet-visible (UV-vis) spectroscopy and some of them are observed in the visible spectral regions. For example, a degassed DMSO solution of cheap and readily available potassium polysulfide $\left(\mathrm{K}_{2} \mathrm{~S}_{\mathrm{x}}\right.$, US $\$ 0.12$ per gram) shows blue color and its steady-state UV-vis absorption spectrum indicates the presence of persistent $\mathrm{S}_{3}{ }^{--}$ ( $\lambda_{\max }$ at $618 \mathrm{~nm}$ with a wide bandwidth ranging from $450 \mathrm{~nm}$ to $\left.800 \mathrm{~nm}\right), \mathrm{S}_{4}{ }^{2-}\left(\lambda_{\max }\right.$ at $436 \mathrm{~nm}$ and $\left.333 \mathrm{~nm}\right)$ and $\mathrm{S}_{3}{ }^{2-}\left(\lambda_{\max }\right.$ at $\left.273 \mathrm{~nm}\right)$ (Fig. 1B). We posited that based on the redox potentials and visible photon absorptions of $\mathrm{S}_{4}{ }^{2-}$ and $\mathrm{S}_{3}{ }^{--}$in their ground state, oxidizible $\mathrm{S}_{4}{ }^{2-}$ could potentially serve as a photo-excited reductant, whereas reducible $\mathrm{S}_{3}{ }^{\cdot-}$ could function as a photo-excited oxidant (4). Therefore, we anticipated that these polysulfide anions could be engaged seamlessly in SET-driven radical-mediated processes in a redox catalytic manifold under visible light irradiation. Herein, we report the use of polysulfide anions $\mathrm{S}_{4}{ }^{2-}$ and $\mathrm{S}_{3}{ }^{--}$as photoredox catalysts for aryl cross-coupling reactions. The reaction design leverages photo-excitation of $\mathrm{S}_{4}{ }^{2-}$ to induce single-electron-reduction of aryl halides having reduction potentials $\left(E_{\text {red }}\right)$ as low as $-2.4 \mathrm{~V}$ (vs SCE). The resulting aryl radicals are engaged in (hetero)biaryl cross coupling, borylation, and hydrogenation in a redox catalytic regime where redox interplay between $\mathrm{S}_{4}{ }^{-} / \mathrm{S}_{4}{ }^{2-}$ and $\mathrm{S}_{3}{ }^{-} / \mathrm{S}_{3}{ }^{2-}$ redox couples enables the redox-neutral catalytic turnover.

At the outset of the project, we explored if the DMSO solution of $\mathrm{K}_{2} \mathrm{~S}_{\mathrm{x}}$ containing $\mathrm{S}_{3}{ }^{\cdot-}$, $\mathrm{S}_{4}{ }^{2-}$ and $\mathrm{S}_{3}{ }^{2-}$ could engage aryl halides in radical coupling reactions under visible light irradiation. We selected to investigate a heterobiaryl coupling of 4'-bromoacetophenone $\left(1, \mathrm{E}_{\text {red }}=-1.89 \mathrm{~V}\right.$ vs SCE) as an electrophore with Nmethylpyrrole (2) as a radical acceptor (16). The current state-of-the-art strategies for such a radical-based aryl cross-coupling leverage highly reducing photo-excited radical anions of polyaromatic hydrocarbons as a photoexcited reductant. In particular, consecutive photoelectron-transfer processes shown by König (17, 18) and electrophotocatalytic strategies detailed by Lambert and Lin (19) and Wickens (20) have successfully generated excited radical anions. Leveraging of readily accessible aminoalkyl radicals for halogen-atom transfer agents was recently proven useful to promote aryl cross-coupling by Leonori (21), while the inherent net-oxidative nature of the process necessitates use of a stoichiometric amount of the oxidant. Our optimization of the reaction conditions revealed that irradiation of blue light $\left(\lambda_{\max }=440 \mathrm{~nm}\right)$ to the mixture of 1 and 2 in the presence of $\mathrm{K}_{2} \mathrm{~S}_{\mathrm{x}}$ (12.5 mol\% per $\mathrm{S}$ atom), potassium carbonate $\left(\mathrm{K}_{2} \mathrm{CO}_{3}, 1.5\right.$ equiv.) and water $\left(\mathrm{H}_{2} \mathrm{O}, 2\right.$ equiv.) in DMSO enabled an efficient coupling to afford heterobiaryl 3 in $86 \%$ yield within 1.5 hours (Fig. 1C). The control experiments indicated that $\mathrm{K}_{2} \mathrm{~S}_{\mathrm{x}}$, irradiation of blue light, and buffer $\left(\mathrm{K}_{2} \mathrm{CO}_{3}\right)$ are all essential for the process, and the reaction is hampered under an air atmosphere (see table $\mathrm{S} 1$ ). The following synergistic catalytic cycle involving $\mathrm{S}_{4}{ }^{2-} / \mathrm{S}_{4}{ }^{--}$and $\mathrm{S}_{3}{ }^{2-} / \mathrm{S}_{3}{ }^{--}$redox couples is proposed (Fig. 1D). Photo-excitation by 440 $\mathrm{nm}$ light endows $\mathrm{S}_{4}{ }^{2-}$ with highly reducing potential in its excited state (22), allowing for single-electronreduction of $\mathbf{1}$ to form arene radical anion $\mathbf{I}$ along with generation of $\mathrm{S}_{4}{ }^{-}$. Single-electron-reduction of reducible $\mathrm{S}_{4}{ }^{--}$by concomitant ground state oxidizable $\mathrm{S}_{3}{ }^{2-}$ allows for regeneration of ground-state $\mathrm{S}_{4}{ }^{2-}$. Meanwhile, the resulting arene radical anion I undergoes mesolysis of the carbon-halogen bond to afford aryl 
radical II (23), that adds onto 2 to form radical intermediate III. Single-electron-oxidation of III by photoexcited $\mathrm{S}_{3}{ }^{--}$followed by deprotonation liberates 3 and ground-state $\mathrm{S}_{3}{ }^{2-}$.

A Redox potentials of $S_{4}^{-}-1 S_{4}{ }^{2-}$ and $S_{3}{ }^{--} / S_{3}{ }^{2-}$ couples

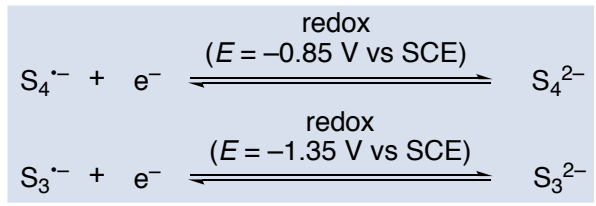

B UV-vis spectrum of potassium polysulfide in DMSO

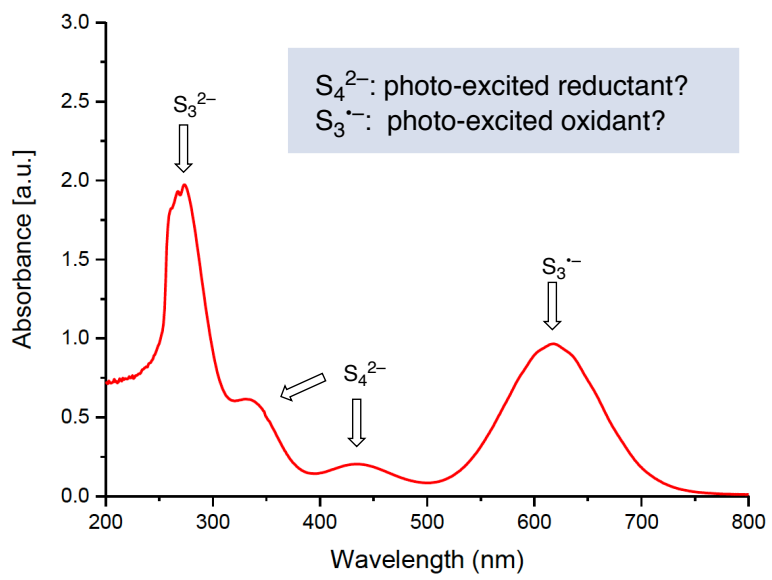

C Optimized heterobiaryl cross coupling of 1 and 2

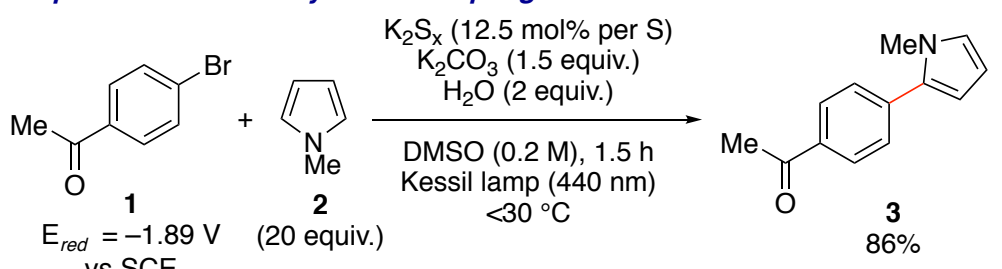

vs SCE

D A proposed catalytic cycle

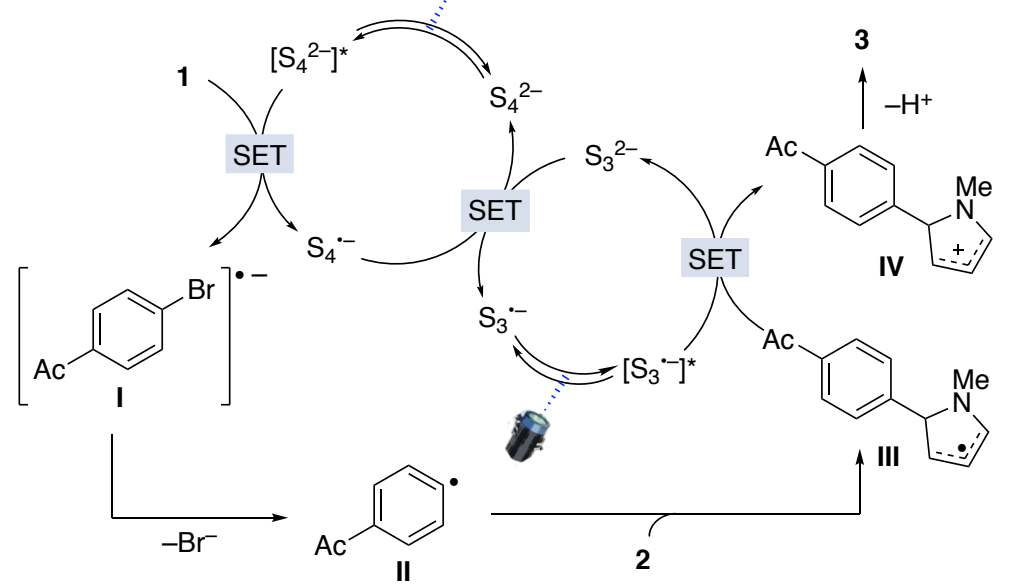

Fig. 1. Reaction development. (A) Redox potentials of $S_{3}{ }^{2-} / S_{3}{ }^{--}$and $S_{4}{ }^{2-} / S_{4}{ }^{--}$couples. (B) UV-vis spectrum of potassium polysulfide $\left(\mathrm{K}_{2} \mathrm{~S}_{\mathrm{x}}\right)$ in DMSO. (C) Optimized reaction conditions catalyzed by $\mathrm{K}_{2} \mathrm{~S}_{\mathrm{x}}: 1$ (0.5 mmol), 2 (20 equiv.), $\mathrm{K}_{2} \mathrm{CO}_{3}$ (1.5 equiv.), $\mathrm{K}_{2} \mathrm{~S}_{x}\left(12.5 \mathrm{~mol} \%\right.$ per S), $\mathrm{H}_{2} \mathrm{O}$ (2 equiv.), DMSO (2.5 mL), $440 \mathrm{~nm}$ light (Kessil lamp), 1.5 hours. (D) $\mathrm{A}$ proposed catalytic cycle. Ac $=$ acetyl.

We next screened the precatalysts of the polysulfide anions in the cross-coupling between 1 and 2 (Fig. 2 A, table S2). Top-down generation of polysulfide anions through reductive fragmentation of octasulfur $\left(\mathrm{S}_{8}\right)(24$, $25)$ in the presence of sodium tert-butoxide $(\mathrm{NaO} t-\mathrm{Bu})$ in DMSO was amenable for the productive crosscoupling. We also found that bottom-up generation of polysulfide anions from mono-sulfide species is suitable for the catalysis. For example, use of dilithium sulfide $\left(\mathrm{Li}_{2} \mathrm{~S}, 10 \mathrm{~mol} \%\right)$ as a precatalyst led to a full conversion of 1 within $2 \mathrm{~h}$ to afford 3 in $88 \%$ yield. Similarly, neutral triisopropylsilylthiol $\left(i-\operatorname{Pr}_{3} \operatorname{SiSH}\right)$, which has commonly been utilized as a hydrogen-atom-transfer catalyst (26), could also perform as a promising precatalyst. These monosulfides neither showed absorption at the visible region nor facilitated the crosscoupling reaction under the dark conditions due to insufficient oxidation potential of monosulfide ions $\left(E_{o x}\right.$ of $\mathrm{S}^{2-}=-0.76 \mathrm{~V}$ vs SCE) (27) (fig. S13-S15). On the other hand, a charge-transfer absorption band was observed from the mixture of 1 and $\mathrm{Li}_{2} \mathrm{~S}$ (Fig. 2B) and irradiation of blue light (440 nm) to a mixture of 1 and $\mathrm{Li}_{2} \mathrm{~S}$ (in 1:1 molar ratio) in DMSO formed acetophenone (4), biaryl 5 and diaryl sulfide $\mathbf{6}$, all of which could be derived 
from the corresponding aryl radical (Fig. 2C, fig. S4). We propose that $\mathrm{Li}_{2} \mathrm{~S}$ triggers the cross-coupling process through the formation of electron-donor-acceptor (EDA) complex $\mathbf{V}$ with $\mathbf{1}$, that induces single-electrontransfer upon irradiation of visible light to produce a radical ion pair $(28,29)$ (Fig. 2D). The resulting radical anion of 1 undergoes cleavage of the $\mathrm{C}$-Br bond to form the aryl radical, whereas a simultaneously formed monosulfide anion radical $\left(\mathrm{S}^{--}\right)$undergoes dimerization to form disulfide dianion $\left(\mathrm{S}_{2}{ }^{2-}\right)$ and its subsequent disproportionation generates the higher order photoredox active polysulfides (30), which promote the photocatalytic turnover further. Interestingly, $i-\mathrm{Pr}_{3} \mathrm{SiSH}$ might initiate the bottom-up formation of polysulfide anions in a different manner. We observed that treatment of $i$ - $\mathrm{Pr}_{3} \mathrm{SiSH}$ with $\mathrm{K}_{2} \mathrm{CO}_{3}$ in DMSO immediately stains the solution blue and the UV-vis absorption spectroscopy unambiguously indicated the generation of polysulfide anions $\left(\mathrm{S}_{3}{ }^{--}, \mathrm{S}_{4}{ }^{2-}\right.$ and $\mathrm{S}_{3}{ }^{2-}$ ) (fig. S16). The nuclear magnetic resonance (NMR) spectroscopy showed the formation of disulfide $\left(i-\mathrm{Pr}_{3} \mathrm{SiS}\right)_{2}$ VI in the solution (fig. S5). Therefore, we postulated that DMSO functions as an oxidant (31) to promote desilylative oligomerization of $i$ - $\mathrm{Pr}_{3} \mathrm{SiSH}$ to the higher order polysulfides via disulfide VI (Fig. 2E). Capability of disulfide VI as the catalyst was ascertained as it performed the productive cross-coupling (table. S2).

A screening of other precatalysts for polysulfide anions

\begin{tabular}{ccc}
\hline Precatalysts & time $(\mathrm{h})$ & yield of $\mathbf{3}(\%)$ \\
\hline $\mathrm{S}_{8}(10 \mathrm{~mol} \%$ / S $)+\mathrm{NaO} t$-Bu $(10 \mathrm{~mol} \%)$ & 3 & 85 \\
$\mathrm{Li}_{2} \mathrm{~S}(10 \mathrm{~mol} \%)$ & 2 & 88 \\
$i-\mathrm{Pr}_{3} \mathrm{SiSH}(10 \mathrm{~mol} \%)$ & 2 & 82 \\
\hline
\end{tabular}

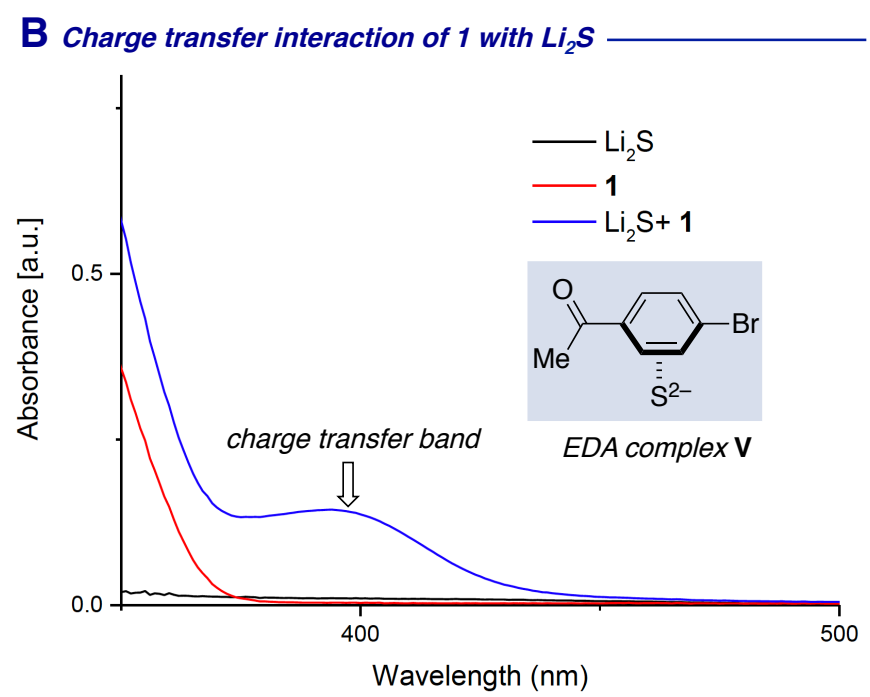

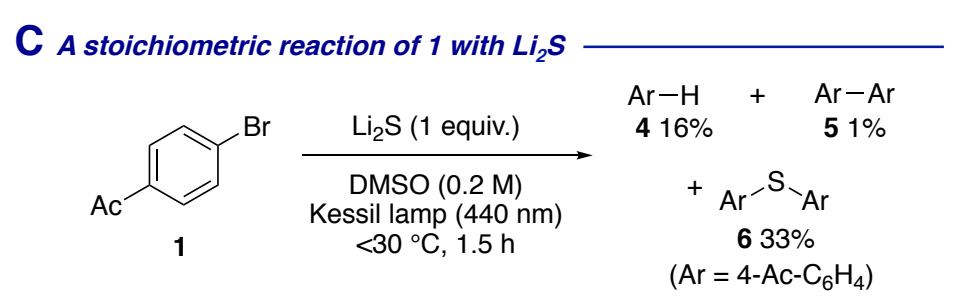

D Bottom-up generation of polysulfide anions from $\mathrm{Li}_{2} \mathrm{~S}$
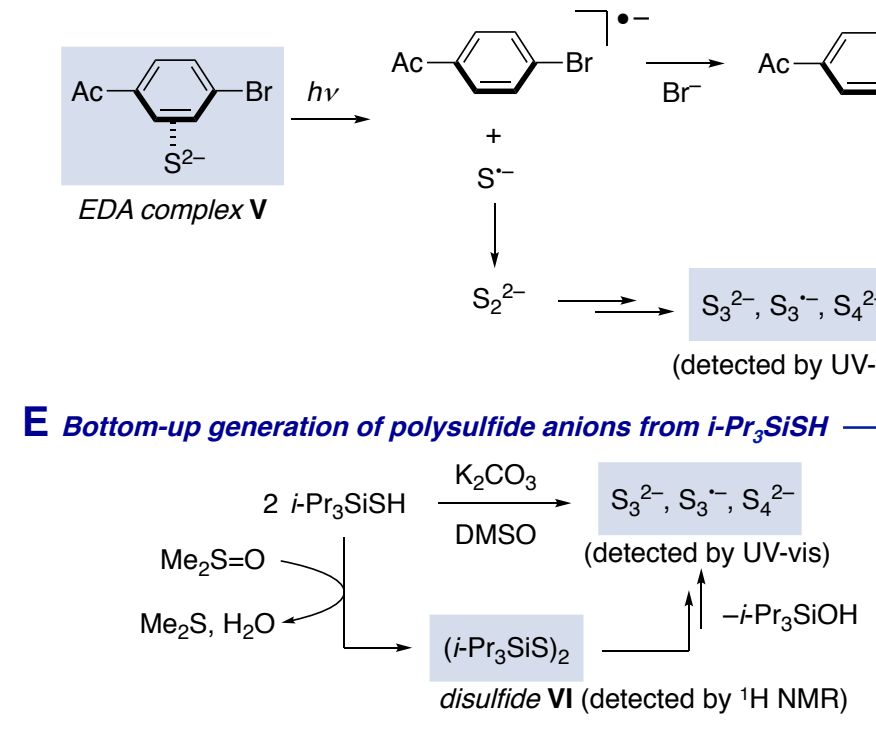

Fig. 2. Investigation of precatalysts. (A) Evaluation of other precatalysts: $1(0.5 \mathrm{mmol}), 2$ (20 equiv.), precatalysts (10 mol\%), $\mathrm{K}_{2} \mathrm{CO}_{3}$ (1.5 equiv.), DMSO (2.5 mL), $440 \mathrm{~nm}$ light (Kessil lamp), $<30{ }^{\circ} \mathrm{C}$. NMR yields of 3 were shown. (B) UV-vis spectra for charge-transfer complex of 4'-bromoacetophenone (1) with dilithium sulfide ( $\left.\mathrm{Li}_{2} \mathrm{~S}\right)$. (C) A stoichiometric reaction of 4'-bromoacetophenone (1) with dilithium sulfide ( $\left.\mathrm{Li}_{2} \mathrm{~S}\right)$ : 1 (0.5 mmol), Li $2 \mathrm{~S}$ (1 equiv.), DMSO (2.5 mL), $440 \mathrm{~nm}$ light (Kessil lamp), 1.5 hours. NMR yields of the products were shown. $\quad$ (D) Bottom-up generation of polysulfide anions from $\mathrm{Li}_{2} \mathrm{~S}$. (E) Bottom-up generation of polysulfide anions from $i-\mathrm{Pr}_{3} \mathrm{SiSH}$. 
We found that this photoredox protocol with polysulfide anions is capable of engaging a wide range of aryl halides for the (hetero)biaryl coupling (Fig. 3A). We first studied the reactivity of 4'-chlolroacetophenone (7), having a reductively inert $\mathrm{C}-\mathrm{Cl}$ bond (32). We observed diminished efficiency in the reaction with $\mathrm{K}_{2} \mathrm{~S}_{\mathrm{x}}(12.5$ $\mathrm{mol} \%$ per $\mathrm{S}$ atom), resulting in premature conversion of 7 (60\%) even after irradiation for $22 \mathrm{~h}$ (table S3). We found that use of $\mathrm{Li}_{2} \mathrm{~S}$ and $i$ - $\mathrm{Pr}_{3} \mathrm{SiSH}$ results in completion of the process within $4 \mathrm{~h}$ to give coupling product 3 in $80 \%$ and $75 \%$ yields, respectively. These outcomes suggested that bottom-up preparation of the polysulfide anions from mono sulfides would provide more productive reactivity especially for reductively recalcitrant aryl halides. The method allows for installation of various polar- $\pi$ electron-withdrawing groups susceptible to reductive reaction conditions, such as ketone (7-10), aldehyde (11-13), nitrile (14) and ester (15). The protocol could successfully engage five-membered ring heteroaryl halides based on furan $(\mathbf{1 6})$, thiophene $(\mathbf{1 7}, \mathbf{1 8})$, and thiazole (19). The chemistry was also extended to functionalize six-membered ring heteroaryl halides such as pyridine (20, 21), quinoline (22) and pyrazine (23). We also found that non-activated aryl halides having a highly negative reduction potential $\left(E_{\text {red }}>-2.4 \mathrm{~V}\right.$ vs SCE) are suitable substrates (24-27). In these cases, employment of $\mathrm{Li}_{2} \mathrm{~S}$ or $i-\mathrm{Pr}_{3} \mathrm{SiSH}(10 \mathrm{~mol} \%)$ as a precatalyst was optimal. However, the reaction of reductively more inert 4-bromoanisole (28) $\left(\mathrm{E}_{\text {red }}=-2.9 \mathrm{~V}\right.$ vs SCE) (33) was found to be sluggish. This protocol was found to be capable in functionalization of nicegoline (29) and indomethacin methyl ester (30) without damaging of other functional groups in these substrates. Finally, we explored if polyhalogenated aromatic substrates could be engaged in chemoselective cross-coupling processes. We were pleased to observe that 2-bromo-4chlorobenzaldehyde (31) was selectively functionalized on the $\mathrm{C}-\mathrm{Br}$ bond. Similarly, the coupling reaction of 4-bromo-2-fluoro-1,1'-biphenyl (32) occurs selectively at the C-Br bond. A more reactive C-I bond (34) could be functionalized selectively in the reactions of methyl 3-bromo-5-iodobenzoate (33) and 1-bromo-4iodobenzene (34). Moreover, 3,5-dibromobenzonitrile (35) was found to undergo single functionalization at one of the C-Br bonds. The scope with respect to the trapping (hetero)arenes was next evaluated (Fig. 3B). Use of $\mathrm{N}-\mathrm{H}$ pyrrole (36) and indole (37) was found to be optimal, while the coupling with thiophene (38) resulted in moderate efficiency. The protocol enables the Minisci type-coupling with pyrazine (39). Electron-rich benzenes $(\mathbf{4 0 , 4 1 )}$ were also found to be compatible as a coupling partner. 

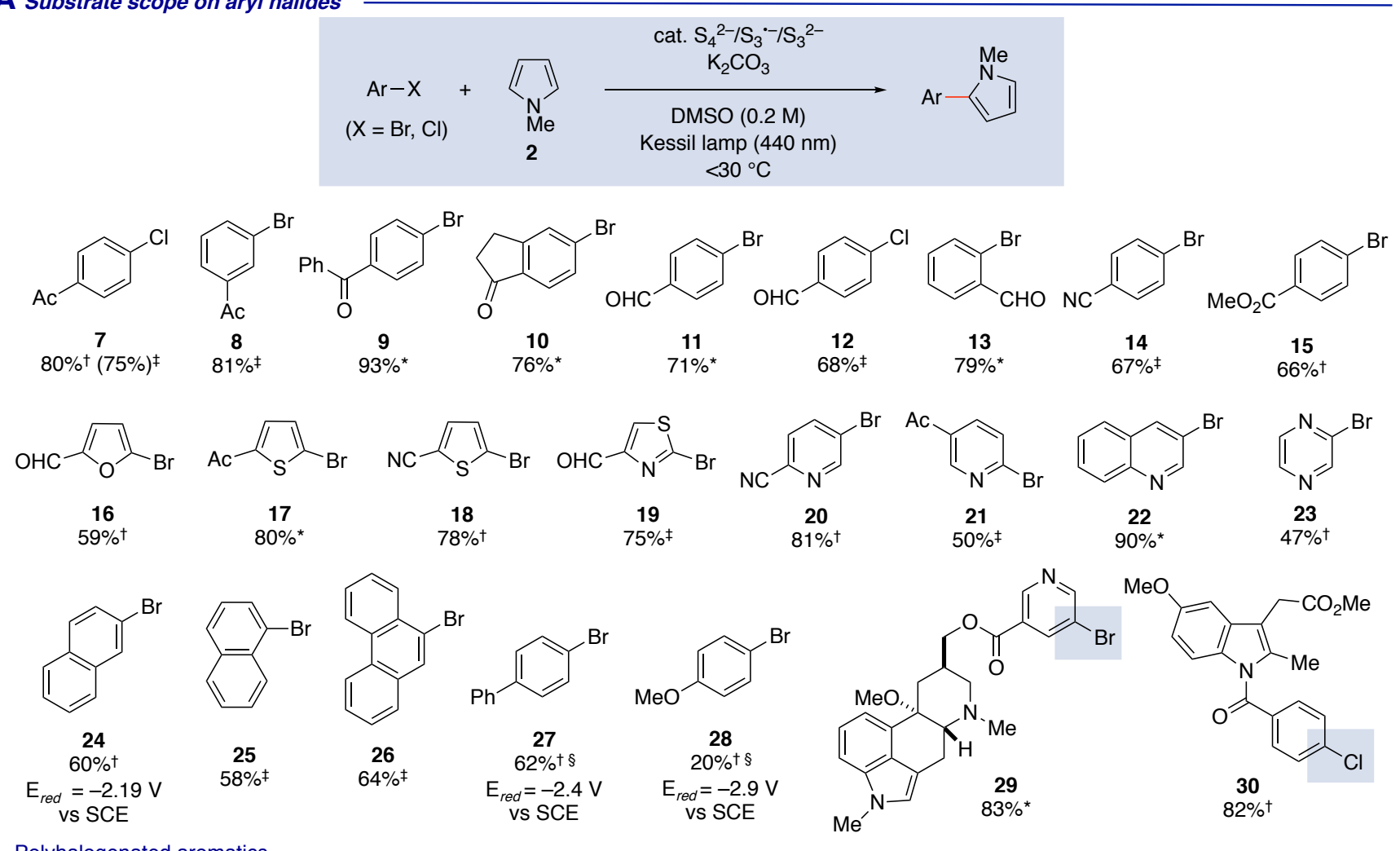

Polyhalogenated aromatics

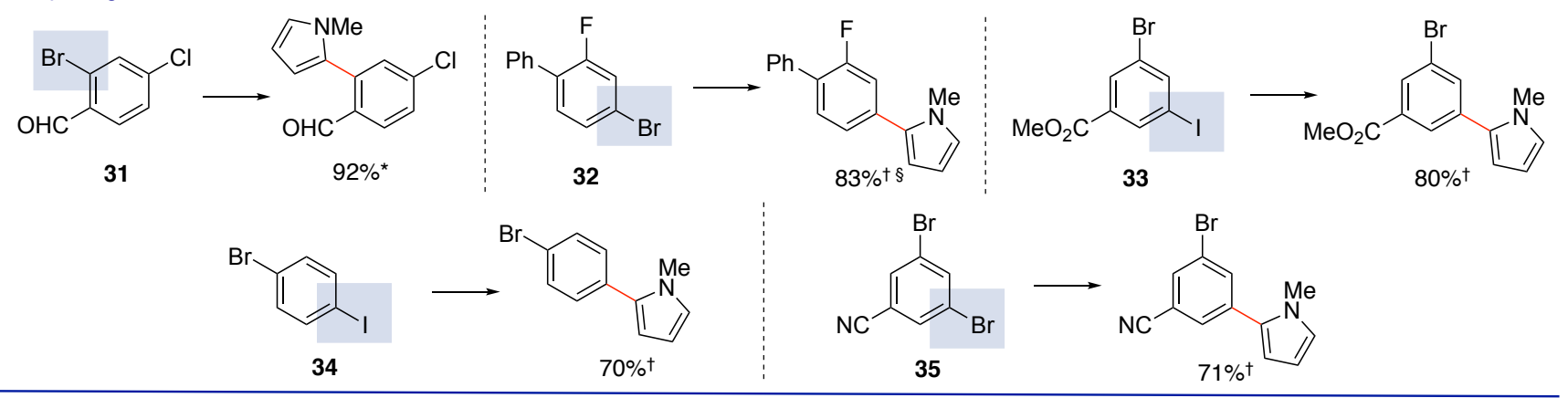

B substrate scope on arene coupling partners

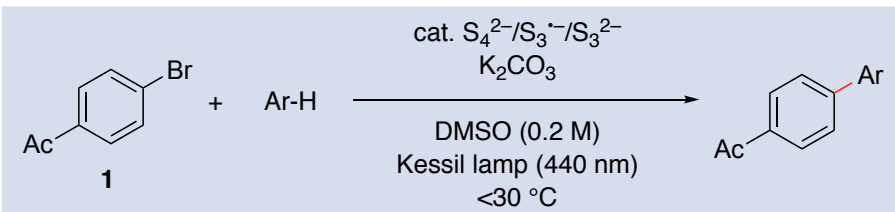

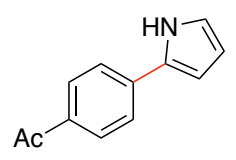

36
$85 \%$ *
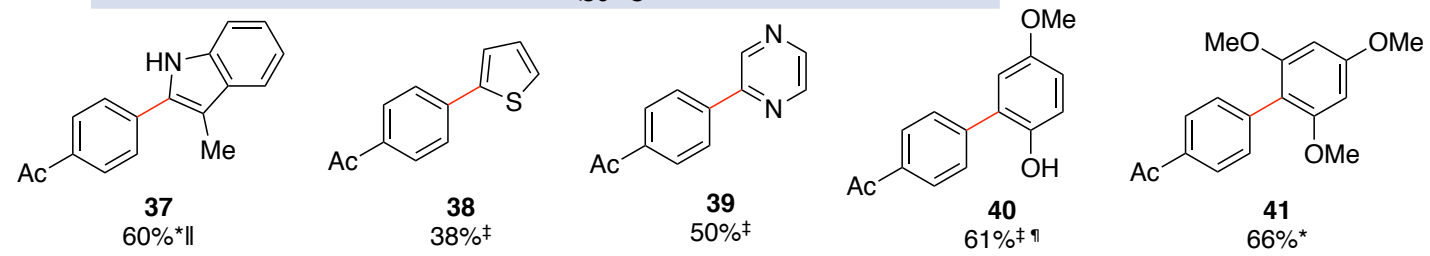

Fig. 3. Reaction scope on (hetero)biaryl cross coupling. (A) Scope of aryl halides. (B) Scope of arene coupling partners: aryl halides $(0.5 \mathrm{mmol})$, coupling partners (20 equiv.), precatalysts (10-20 mol\%), $\mathrm{K}_{2} \mathrm{CO}_{3}(1.5$ equiv.), DMSO $(2.5 \mathrm{~mL}), 440 \mathrm{~nm}$ light (Kessil lamp), $<30{ }^{\circ} \mathrm{C}$. Isolated yields of the products were recorded. * Precatalyst: $\mathrm{K}_{2} \mathrm{~S}_{\mathrm{x}}(12.5$ mol\% per S) with $\mathrm{H}_{2} \mathrm{O}$ (2 equiv.). † Precatalyst: $\mathrm{Li}_{2} \mathrm{~S}$ (10 mol\%). ‡ Precatalyst: $i-\mathrm{Pr}_{3} \mathrm{SiSH}(10 \mathrm{~mol} \%)$. $\$ 390 \mathrm{~nm}$ light. ॥ 10 equiv of 3-methylindole was used. If The reaction was run with 5 equiv of 4-methoxyphenol and 5 equiv of $\mathrm{NaOt}$-Bu. 
Synthetic utility of this polysulfide anions-based photoredox catalysis was further extended to the dehaloborylation reaction by employing bis(pinacolato)diboron $\left(\mathrm{B}_{2} \mathrm{pin}_{2}\right)$ as the radical trapping reagents (Fig. 4A, table S4) (35). Optimization for the borylation of 4'-bromoacetophenone (1) with $\mathrm{K}_{2} \mathrm{~S}_{\mathrm{x}}$ as a precatalyst led to the identification of tetramethylguanidine (TMG) and acetonitrile $\left(\mathrm{CH}_{3} \mathrm{CN}\right)$ as the optimal base and solvent, respectively, delivering pinacol arylboronate 42 in $84 \%$ yield within $1.5 \mathrm{~h}$. This protocol was found to be applicable to the borylation of various functionalized haloarenes (43-47). We also found that the protocol is amenable to hydrodebromination of 1 using diisopropylethylamine $\left(i-\operatorname{Pr}_{2} \mathrm{NEt}\right)$ as a hydrogen donor, providing acetophenone (4) in 96\% yield within 2 hours (Fig. 4B, table S5) (36). The identified reaction conditions were capable of reductive radical cyclization of $\mathbf{4 8}$ to dihydrobenzofuran 49 and hydrodeiodination of secondary alkyl iodide $\mathbf{5 0}$ to $\mathbf{5 1}$.

These batch photo-redox processes driven by the polysulfide anions stimulated us to explore the scalability of the heterobiaryl cross-coupling and borylation in flow (Fig. 4C). The cross-coupling between 1 and 2 was efficiently promoted in a homogeneous system using $i$ - $\mathrm{Pr}_{3} \mathrm{SiSH}$ as a precatalyst and tetramethylguanidine (TMG) as a base in an operationally simple micro-tubing continuous-flow reactor (37) (fig. S8). The desired product 3 was delivered at $1.75 \mathrm{~g} /$ hour production rate (78\%) with $30 \mathrm{mins}$ as residence time. The debromo-borylation of $\mathbf{1}$ could also be performed in the same flow reactor to afford 42 at 6.9 g/hour production rate $(83 \%)$ with 20 mins as residence time.

The polysulfide anions-based photoredox catalysis presented herein conveniently engages a wide range of aryl halides in productive cross-coupling chemistry. We anticipate that the broad scope with wide functional group compatibility, operational simplicity and scalability in flow would bring useful and practical applications of this strategy in various fields.

Acknowledgment: We thank H. S. Soo, R. Kinjo, J. M. Hawkins, T. Knauber, F. L. Levesque, L. Edwards and J.-P. Krieger for helpful discussion. We acknowledge financial support was provided by Pharma Innovation Programme Singapore (A*STAR, SERC A19B3a0014) and Nanyang Technological University, Singapore as well as National University of Singapore.

Competing interest: The authors declare no competing interest. 


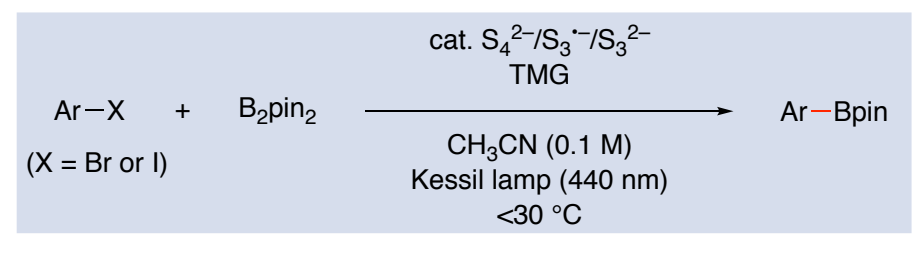

(from 1)

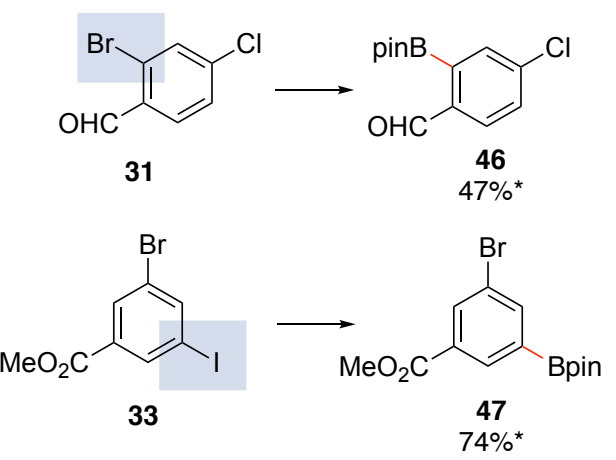

B Hydrodehalogenation
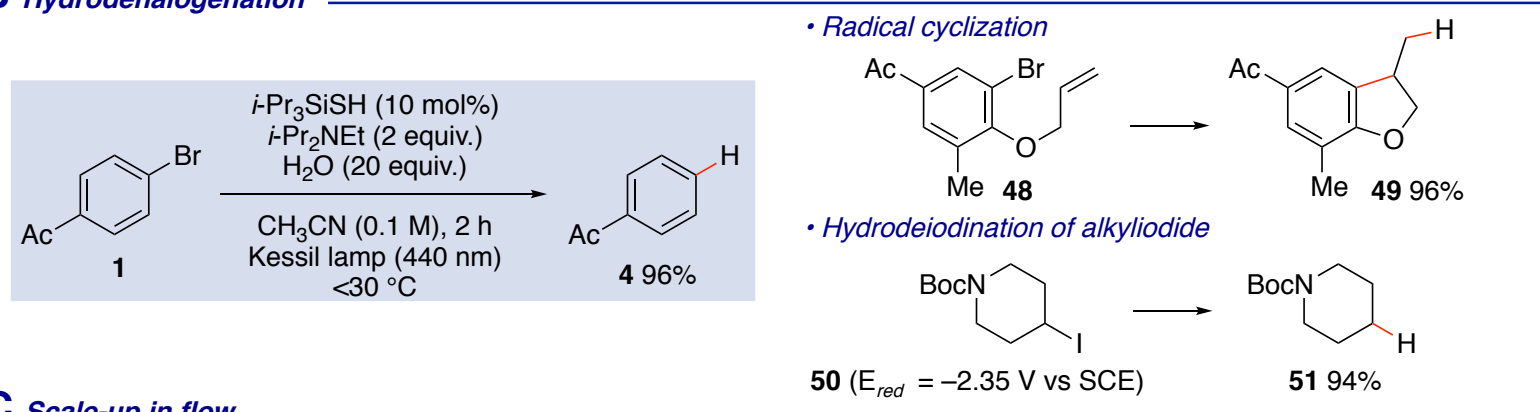

Scale-up in flow
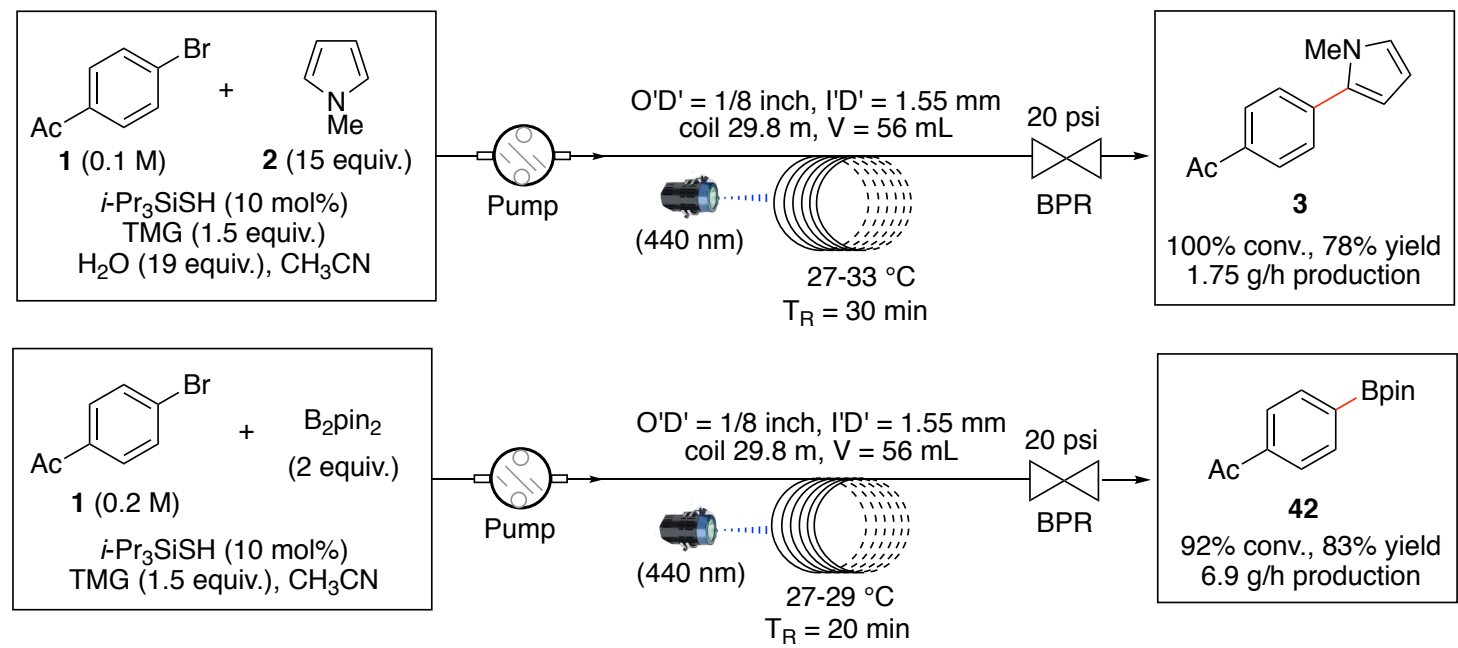

Fig. 4. Application to borylation, hydrogehalogenation and scale-up in flow. (A) Borylation: substrate $(0.5 \mathrm{mmol})$, $\mathrm{B}_{2}$ pin 2 (2 equiv.), precatalysts (10-12.5 mol\%), TMG (1.5 equiv.), $\mathrm{CH}_{3} \mathrm{CN}(5 \mathrm{~mL}), 440 \mathrm{~nm}$ light (Kessil lamp), $<30^{\circ} \mathrm{C}$. (B) Hydrodehalogenation: substrate (0.5 mmol), i- $\mathrm{Pr}_{2} \mathrm{NEt}$ (2 equiv.), $i-\mathrm{Pr}_{3} \mathrm{SiSH}$ (10 mol\%), $\mathrm{H}_{2} \mathrm{O}$ (20 equiv.), $\mathrm{CH}_{3} \mathrm{CN}(5 \mathrm{~mL})$, $440 \mathrm{~nm}$ light (Kessil lamp), $<30^{\circ} \mathrm{C}$. Boc = tert-butoxycarbonyl. (C) Scale up of heterobiaryl cross-coupling and borylation in flow. O'D' = outer diameter; I' $\mathrm{D}$ ' = inner diameter; $\mathrm{T}_{\mathrm{R}}=$ residence time; $\mathrm{V}=$ volume of the micro-tubing reactor; $\mathrm{BPR}=$ back pressure regulator. ${ }^{*}$ Precatalyst: $\mathrm{K}_{2} \mathrm{~S}_{\mathrm{x}}\left(12.5 \mathrm{~mol} \%\right.$ per $\mathrm{S}$ ) with $\mathrm{H}_{2} \mathrm{O}$ (2 equiv.). † Precatalyst: $i-\mathrm{Pr}_{3} \mathrm{SiSH}(10 \mathrm{~mol} \%)$. 


\section{References and Notes:}

1. R. C. McAtee, E. J. McClain, C. R. J. Stephenson, Illuminating photoredox catalysis, Trends Chem. 1, 111125 (2019).

2. J. Twilton, C. C. Le, P. Zhang, M. H. Shaw, R. W. Evans, D. W. C. MacMillan, The merger of transition metal and photocatalysis. Nat. Rev. Chem. 1, 0052 (2017).

3. M. H. Shaw, J. Twilton, D. W. C. MacMillan, Photoredox catalysis in organic chemistry. J. Org. Chem. 81, 6898-6926 (2016).

4. N. A. Romero, D. A. Nicewicz, Organic photoredox catalysis. Chem. Rev. 116, 10075-10166 (2016).

5. D. M. Schultz, T. P. Yoon, Solar synthesis: prospects in visible light photocatalysis. Science 343, 1239176 (2014).

6. I. Ghosh, J. Khamrai, A. Savateev, N. Shlapakov, M. Antonietti, B. König, Organic semiconductor photocatalyst can bifunctionalize arenes and heteroarenes. Science 365, 360-366 (2019).

7. Z. Zhang, K. Edme, S. Lian, E. A. Weiss, Enhancing the rate of quantum-dot-photocatalyzed carbon-carbon coupling by tuning the composition of the dot's ligand shell. J. Am. Chem. Soc. 139, 4246-4249 (2017).

8. J. A. Caputo, L. C. Frenette, N. Zhao, K. L. Sowers, T. D. Krauss, D. J. Weix, General and efficient C-C bond forming photoredox catalysis with semiconductor quantum dots. J. Am. Chem. Soc. 139, 4250-4253 (2017).

9. R. Steudel, T. Chivers, The role of polysulfide dianions and radical anions in the chemical, physical and biological sciences, including sulfur-based batteries. Chem. Soc. Rev. 48, 3279-3319 (2019).

10. T. Chivers, P. J. W. Elder, Ubiquitous trisulfur radical anion: fundamentals and applications in materials science, electrochemistry, analytical chemistry and geochemistry. Chem. Soc. Rev. 42, 5996-6005 (2013).

11. J. Scheers, S. Fantini, P. Johansson, A review of electrolytes for lithium-sulphur batteries. J. Power Sources 255, 204-218 (2014).

12. Q. Zou, Y.-C. Lu, Solvent-dictated lithium sulfur redox reactions: an operando UV-vis spectroscopic study. J. Phys. Chem. Lett. 7, 1518-1525 (2016).

13. B.-S. Kim, S.-M. Park, In situ spectroelectrochemical studies on the reduction of sulfur in dimethyl sulfoxide solutions. J. Electrochem. Soc. 140, 115-122 (1993).

14. P. Leghie, J.-P. Lelieur, E. Levillain, Comments on the mechanism of the electrochemical reduction of sulphur in dimethylformamide. Electrochem. Commun. 4, 406-411 (2002).

15. H. G. Roth, N. A. Romero, D. A. Nicewicz, Experimental and calculated electrochemical potentials of common organic molecules for applications to single-electron redox chemistry. Synlett 27, 714-723 (2016).

16. I. Ghosh, L. Marzo, A. Das, R. Shaikh, B. König, Visible light mediated photoredox catalytic arylation reactions. Acc. Chem. Res. 49, 1566-1577 (2016).

17. I. Ghosh, T. Ghosh, J. I. Bardagi, B. König, Reduction of aryl halides by consecutive visible light-induced electron transfer processes. Science 346, 725-728 (2014).

18. I. Ghosh, B. König, Chromoselective photocatalysis: controlled bond activation through light-color regulation of redox potentials. Angew. Chem. Int. Ed. 55, 7676-7679 (2016).

19. H. Kim, H. Kim, T. H. Lambert, S. Lin, Reductive electrophotocatalysis: merging electricity and light to achieve extreme reduction potentials. J. Am. Chem. Soc. 142, 2087-2092 (2020). 
20. N. G. W. Cowper, C. P. Chernowsky, O. P. Williams, Z. K. Wickens, Potent reductants via electron-primed photoredox catalysis: unlocking aryl chlorides for radical coupling. J. Am. Chem. Soc. 142, 2093-2099 (2020).

21. T. Constantin, M. Zanini, A. Regni, N. S. Sheikh, F. Juliá, D. Leonori, Aminoalkyl radicals as halogen-atom transfer agents for activation of alkyl and aryl halides. Science 367, 1021-1026 (2020).

22. See the supplementary materials for more information.

23. N. Takeda, P. V. Poliakov, A. R. Cook, J. R. Miller, Faster dissociation: measured rates and computed effects on barriers in aryl halide radical anions. J. Am. Chem. Soc. 126, 4301-4309 (2004).

24. T. Chivers, Ubiquitous trisulphur radical ion $\mathrm{S}_{3}{ }^{--}$. Nature 252, 32-33 (1974).

25. G. Zhang, H. Yi, H. Chen, C. Bian, C. Liu, A. Lei, Trisulfur radical anion as the key intermediate for the synthesis of thiophene via the interaction between elemental sulfur and $\mathrm{NaOtBu}$. Org. Lett. 16, 6156-6159 (2014).

26. A. Breder, C. Depken, Light-driven single-electron transfer processes as an enabling principle in sulfur and selenium multicatalysis. Angew. Chem. Int. Ed. 58, 17130-17147 (2019).

27. J. A. Dean, “Langes Handbook of Chemistry, 15 $5^{\text {th }}$ Ed.”, McGraw-Hill, Inc., New York (1992).

28. B. Liu, C.-H. Lim, G. M. Miyake, Visible-light-promoted C-S cross-coupling via intermolecular charge transfer. J. Am. Chem. Soc. 139, 13616-13619 (2017).

29. G. E. M. Crisenza, D. Mazzarella, P. Melchiorre, Synthetic methods driven by the photoactivity of electron donor-acceptor complexes. J. Am. Chem. Soc. 142, $5461-5476$ (2020).

30. T. S. Bailey, H. A. Henthorn, M. D. Pluth, The intersection of $\mathrm{NO}$ and $\mathrm{H}_{2} \mathrm{~S}$ : persulfides generate $\mathrm{NO}$ from nitrite through polysulfide formation. Inorg. Chem. 55, 12618-12625 (2016).

31. T. J. Wallace, J. J. Mahon, Reactions of thiols with sulfoxides. II. Kinetics and mechanistic implications. $J$. Am. Chem. Soc. 86, 4099-4103 (1964).

32. C. P. Andrieux, C. Blocman, J. M. Dumas-Bouchiat, F. M'Halla, J. M. Savéant, Determination of the lifetimes of unstable ion radicals by homogeneous redox catalysis of electrochemical reactions. Application to the reduction of aromatic halides. J. Am. Chem. Soc. 102, 3806-3813 (1980).

33. I. A. MacKenzie, L. Wang, N. P. R. Onuska, O. F. Williams, K. Begam, A. M. Moran, B. D. Dunietz, D. A. Nicewicz, Discovery and characterization of an acridine radical photoreductant. Nature 580, 76-80 (2020).

34. L. Pause, M. Robert, J.-M. Savéant, Can single-electron transfer break an aromatic carbon-heteroatom bond in one step? A novel example of transition between stepwise and concerted mechanisms in the reduction of aromatic iodides. J. Am. Chem. Soc. 121, 7158-7159 (1999).

35. K. Chen, L. Wang, G. Meng, P. Li, Recent advances in transition-metal-free aryl C-B bond formation. Synthesis 49, 4719-4730 (2017).

36. J. D. Nguyne, E. M. D’Amato, J. M. R. Narayanam, C. R. J. Stephenson, Engaging unactivated alkyl, alkenyl and aryl iodides in visible-light-mediated free radical reactions. Nat. Chem. 4, 854-859 (2012).

37. D. Cambié, C. Bottecchia, N. J. W. Straathof, V. Hessel, T. Noël, Applications of continuous-flow photochemistry in organic synthesis, material science, and water treatment. Chem. Rev. 116, 10276-10341 (2016). 